\title{
Détermination Du Seuil De Viabilité De La Position Extérieure Au Maroc: Evaluation Empirique
}

\author{
Abdellali Fadlallah \\ Professeur Assistant, INSEA, Rabat, Maroc \\ Zakaria Chakhat \\ Doctorant, Université Hassan $1^{\text {er }}$ Settat, Maroc
}

Doi: 10.19044/esj.2018.v14n7p32 URL:http://dx.doi.org/10.19044/esj.2018.v14n7p32

\begin{abstract}
This paper focuses on addressing question on the sustainability of the Moroccan current account. The purpose of this study is to examine theoretical and empirical determinants of the behaviour of the current account deficit in order to apprehend economic policy decisions. An econometric study was conducted in this sense using a vector error-correction model (VECM) during the period (2004Q1-2013Q4). This technique enabled the capture of the longterm relationship. the impact of explanatory variables on the current account was also examined. The examination of the sustainability threshold calculated by the Reisena method revealed that signs of unsustainability have emerged since 2008, which is in line with the results of MFR. However, it is only in 2011 that the current account exhibits deficits that largely exceed the optimal threshold, exposing the national economy to greater vulnerability.
\end{abstract}

Keywords: Deficit of the current account, Viability, VECM

\section{Résumé}

Le présent document traite la problématique de la soutenabilité du compte courant marocain. L'ojectif est l'examen théorique et empirique des différents déterminants du comportement du déficit courant pour mieux appréhender les décisions de politique économique. Une étude économétrique a été réalisé dans ce sens, où on a opté pour une modélisation de vecteur à correction d'erreur VECM dural la période (2004Q1-2013Q4). Cette technique a permis de capter la relation du long terme, ensuite l'étude a porté sur l'impact des variables explicatives sur le compte courant. L'examen du seuil de soutenabilité calculé par la méthode de Reisena a montré conformément aux résultats de MFR que les signes d'insoutenabilité apparaissent depuis 2008. Cependant, ce n'est qu'en 2011 que le compte courant affiche des déficits qui dépassent largement le seuil optimal exposant 
l'économie nationale à une plus grande vulnérabilité.

Mots Clés: Déficit du compte courant, viabilité, modèle à correction d'erreur

\section{Introduction}

Le processus d'intégration du Maroc dans l'économie mondiale exige une grande disposition à l'adaptation aux normes standards régulant le marché international. Il est donc nécessaire pour le Maroc de préparer une plateforme adéquate afin d'assouplir la transition vers l'ouverture et limiter les effets néfastes qui pourraient en résulter.

L'ouverture économique du Maroc a suscité la mise en place de plusieurs politiques notamment monétaires. En effet, pour accompagner cette réforme stratégique, les politiques publiques ont été appelées à répondre à une exigence précise à savoir la mise en place des mesures pour pouvoir mettre à niveau les secteurs économiques principalement par :

$>\quad$ La libéralisation des investissements étrangers au Maroc et des financements extérieurs pour les entreprises résidentes au début des années 90 ;

L'adhésion à l'Article VIII des Statuts du FMI en 1993 qui consacre la libéralisation des opérations courantes ;

$>\quad$ La mise en place du marché des changes en 1996 qui a mis fin notamment au monopole de la banque centrale en matière de gestion des devises ;

$>\quad$ La libéralisation en 2002 des placements à l'étranger des banques résidentes.

Ce choix a été couronné par la signature d'un éventail d'accords bilatéraux ou multilatéraux qui donnent droit à des réductions tarifaires ou qui préconisent des arrangements commerciaux préférentiels. Ces accords s'inscrivent dans le cadre des efforts déployés par le Maroc visant à renforcer son ancrage à un environnement régional et international en profonde mutation. Outre son adhésion à l'OMC en janvier 1995, le Maroc a conclu des accords de libre échange (ALE) avec l'Union européenne (1996), la Zone arabe de libre-échange (1998), l'AELE (2000), l'Accord d'Agadir (2001), la Turquie (2004) et les Etats-Unis (2005).

Cet élan d'ouverture, qui vise une meilleure intégration du Maroc dans l'économie mondiale, doit s'accompagner d'un renforcement du suivi et de la supervision de l'évolution de la situation extérieure du Maroc. Cette situation est reflétée au niveau de la balance des paiements qui est un état statistique qui relate les flux économiques et financiers entre les résidents et les non-résidents.

Plus particulièrement, le compte courant de la balance des paiements est un indicateur incontournable pour les décideurs dans l'analyse de la 
situation économique d'un pays. En effet, le compte courant reflète, d'une part, les transactions sur les biens et services entre les résidents et les non-résidents.

De plus, puisque le compte courant détermine l'évolution dans le temps du stock des engagements extérieurs nets, il renseigne, d'autre part, sur les décisions inter-temporelles des résidents et des non-résidents par rapport notamment à l'épargne et l'investissement. De ce fait, il est important de chercher à expliquer l'évolution passée du compte courant de la balance des paiements et d'évaluer sa soutenabilité à moyen terme afin d'éclairer, quand c'est nécessaire, les actions en la matière.

Le compte courant marocain a été confronté à plusieurs chocs pendant la dernière décennie après avoir inscrit de bonnes performances 15 ans auparavant (avant 2008), exposant ses principales composantes à de sérieux déséquilibres. La conjoncture internationale n'a pas manqué d'aggraver le déficit courant, notamment la récession qui a frappé ses pays partenaires, la hausse des cours du pétrole et la hausse des prix des produits alimentaires.

Ces chocs se sont conjugués à d'autres facteurs internes dont on cite : la rigidité du taux de change et le système de subventions. En effet, les déficits causés par les déséquilibres internes sont plus graves et rendent la balance des paiements plus vulnérable, alors que les déséquilibres externes mènent vers des crises de devises. Dès lors, la question de la soutenabilité du compte courant devient une préoccupation majeure pour évaluer la persistance du déficit et prévenir les ajustements de la politique économique à opérer.

Pour certains chercheurs à l'instar de (Summers 1996), un déficit courant dépassant $5 \%$ du PIB est jugé non-soutenable. Ce déficit devient plus critique lorsqu'il est financé par la dette extérieure ou par l'épuisement des réserves de change. Dans ce sens, la compréhension des déterminants du compte courant, aussi bien que l'analyse de la structure du financement du déficit sont cruciales quant à l'étude de la vulnérabilité du compte courant.

L'objet de cette étude est le calcul du seuil de la soutenabilité du compte courant en se basant sur les études de Milesi-Ferretti et Razin (1996) ainsi que la méthode de Reisen dans la détermination du seuil au moyen terme. Le reste du document est organisé en trois axes. Dans le premier axe on expose une analyse du modèle théorique utilisé pour évaluer la soutenabilité de la Balance des paiements, le deuxième axe exposera le seuil optimal par la méthode Reisen et dernier lieu nous procèderons à la détermination empirique de la soutenabilité la balance des paiements au Maroc.

\section{Le modèle théorique du MFR}

Diverses approches sont utilisées pour analyser la soutenabilité du déficit courant de la balance des paiements. La première, est une méthode comptable, a été notamment appliquée par Husted (1992), Wu, Fountas et Chen (1996). La deuxième méthode repose sur l'approche inter-temporelle 
optimale, elle a été développée par Obstfeld et Rogoff (1995) et qui mesure la capacité d'un pays à dégager suffisamment de surplus commerciaux dans le futur pour rembourser sa dette actuelle.

En effet, en cas de déficit courant, l'économie se retrouve dans l'obligation de le financer par l'accumulation des engagements extérieurs dont il faut assurer le remboursement dans le futur. Toutefois, la principale limite de ces deux approches réside dans la capacité de rembourser, sans tenir compte de la volonté de le faire. En effet, selon l'approche MFR, même lorsque les excédents commerciaux futurs sont suffisants pour couvrir le paiement du service de la dette extérieure future, il pourrait être politiquement impossible de le faire au détriment des dépenses intérieures.

En plus, cette solvabilité se base sur l'hypothèse selon laquelle les créanciers internationaux sont disposés à prêter aux pays en besoin de financement. L'approche MFR est limitée par le réalisme de cette hypothèse, principalement en cas des incertitudes sur la capacité des pays débiteurs à honorer leurs engagements. Milesi-Ferretti et Razin recourent à une équation comptable pour expliquer le sens de la solvabilité inter temporelle, avec le rôle spécial du taux de croissance économique réelle $(\gamma)$, le taux d'intérêt réel de la dette extérieure $(r)$, et taux le taux de change $(\varepsilon)$. On définit la solvabilité intertemporelle suivant les conditions dans lesquelles, le pays comme une entité et tout agent économique y compris le gouvernement obéissent respectivement à la contrainte budgétaire. Le comportement du compte courant est défini comme suit :

$C A D_{t}=F_{t}-F_{t-1}=Y_{t}+r F_{t}-C_{t}-I_{t}-G_{t}$ (1)

Avec : $F_{t}$ : le stock des actifs étrangers, $\mathrm{r}$ : le taux d'intérêt réel (supposé constant) et $C A D_{t}$ : le déficit du compte courant, $\mathrm{I}_{t}$ : le ration des investissement, $\mathrm{C}_{\mathrm{t}}$ : Demande des ménages, $\mathrm{G}_{\mathrm{t}}$ : Consommation publique

La condition de solvabilité est limitée puisqu'elle n'impose aucune structure sur les variables. On peut par contre dériver une condition simple de solvabilité sous l'hypothèse que les agrégats macroéconomiques déflatés par le PIB sont constants, aussi bien que le taux d'intérêt et le taux de change. On suppose que l'économie domestique croit à un taux donné noté $\gamma$, inférieur à $\mathrm{r}^{*}$. On peut réécrire l'identité (1) comme suit :

$s_{t} p_{t}^{*} F_{t}-s_{t} p_{t-1}^{*} F_{t}=p_{t}\left(Y_{t}-C_{t}-G_{t}-I_{t}\right)+i_{t}^{*} s_{t} p_{t-1} F_{t-1}$

Avec: $s_{t}$ : taux de change nominal, $p_{t}$ : déflateur du PIB domestique, $p_{t}^{*}$ : déflateur du PIB étranger et $i_{t}^{*}$ : taux d'intérêt nominal

On divise les deux côtés par le PIB nominal $\left(Y_{t} p_{t}\right)$ domestique, en réarrangeant l'équation (2) sachant que le taux de change réel est égale à $p_{t} / p_{t}^{*} s_{t}$ on obtient :

$$
\left.f_{t}-f_{t-1}=\left[\boldsymbol{r}^{*}-\varepsilon_{t}-\gamma_{t}\right) \boldsymbol{f}_{t}+\boldsymbol{t} \boldsymbol{b}_{t}\right] \frac{1}{\left(1+\gamma_{t}\right)\left(1+\varepsilon_{t}\right)}
$$


$\varepsilon_{t}:$ Variation du taux de change réel, $t b_{t}$ Balance commerciale

Cette équation suppose que le changement du ratio des actifs étrangers par rapport au PIB est conduit par la balance commerciale et la dynamique de la dette qui est positivement liée au taux d'intérêt réel et négativement liée au taux de croissance du PIB domestique et le taux de change réel (TCR).

$\mathrm{Si}$ on considère que l'économie est en équilibre stable, les ratios de la consommation, l'investissement et le stock des actifs étrangers par rapport au PIB sont constants. A partir de cette équation calculée à l'équilibre stable, on peut obtenir le niveau du surplus de la balance commerciale qu'un pays doit générer pour garder le ratio de la dette par rapport au PIB constant. A cet effet on a: $\mathrm{f}_{\mathrm{t}}=\mathrm{f}_{\mathrm{t}-1}=\mathrm{f}$ De l'équation (3) on obtient : $\quad t b=1-c-$ $i-g=-f\left(r^{*}-\varepsilon-\gamma\right)$

La première partie de l'équation montre le fait que l'économie doit être dans un état d'équilibre pour stabiliser la dette par rapport au PIB et correspondre à une balance commerciale viable. La dernière partie de l'expression indique le rôle joué par la valeur moyenne des taux d'intérêt mondiaux, la croissance intérieure et la tendance à long terme du taux de change réel dans la détermination des transferts de ressources nécessaires pour maintenir la viabilité de la dette publique. La condition (4) indique que l'absorption à long terme de l'économie peut être supérieure à son revenu seulement si l'économie est un créancier net.

Doisy et Hervé (2003) ont modifié cette formule afin de tenir compte du fait que plusieurs pays financent leur déficit extérieur par un instrument non créateur de la dette comme les IDE. Cela dit il ne faut pas croire que c'est une forme de financement absolument sans risque. Pourtant leur volatilité est beaucoup plus faible que celle des autres formes. Si les IDE sont pris en compte, le compte courant soutenable en pourcentage du PIB peut s'écrire comme suit :

$C A D=f\left(r^{*}-\varepsilon-\gamma\right)-i d e$

Avec ide : le ratio des investissements directs à l'étranger par rapport au PIB

\section{Le seuil optimal de soutenabilité par la méthode Reisen}

Cette approche est développée par Reisen (1996) et Steiner et Losada (1996) en se basant sur une fonction conventionnelle de la dynamique de la dette extérieure pour analyser la solvabilité inter- temporelle du long terme.

Considérons d'abord une économies table, avec des engagements comme une fraction du $\mathrm{PIB}$ du pays que les étrangers sont prêts à tenir en équilibre et qu'on désigne par f. A l'équilibre, c'est à dire avec f gardé constant, le pays accumule des engagements nets, qui sont égales au déficit du compte courant CAD plus l'accumulation nette de réserves internationales FX, tous les deux en tant que fraction du PIB, en proportion du taux croissance du PIB à 
long terme. On le représente par l'équation (6): $C A D+\triangle F X=$ $\gamma f$

La croissance du PIB exerce deux effets indirects sur l'état stable du compte courant qui est compatible avec un ratio stable de la dette au PIB. En premier lieu, le niveau des réserves internationales souhaité augmente lorsque l'économie est en expansion. La littérature a identifié un facteur déterminant dans la demande des réserves de change, il s'agit de la croissance annuelle réelle des importations dénotées par $\eta$. La variation du ratio des réserves de change par rapport au PIB peut être écrite comme suit :

$$
\Delta F X=(1+\eta) /(1+\gamma) F X-F X
$$

En incorporant (6) en (7) on obtient

$$
\boldsymbol{\gamma} \boldsymbol{f}=\boldsymbol{C A D}+(\boldsymbol{\eta}+\boldsymbol{\gamma}) /(\boldsymbol{1}+\boldsymbol{\gamma}) \boldsymbol{F X}
$$

Un deuxième canal par lequel la croissance du PIB impacte indirectement la dynamique de la dette est l'effet Balassa-Samuelson, à moyen terme, la croissance relative conduit à une appréciation réelle du taux de change, en raison principalement de l'évolution des écarts de productivité entre les marchandises échangeables et non-échangeables dans l'économie nationale et dans le reste du monde. La variation du taux de change $(\varepsilon)$, réduit le ratio de la dette par rapport au PIB et le ratio des réserves de devises, de sorte que l'équation (8) devient :

$$
(\boldsymbol{\gamma}+\boldsymbol{\varepsilon}) \boldsymbol{f}=\boldsymbol{C A D}+[(\boldsymbol{\eta}+\boldsymbol{\varepsilon}-\boldsymbol{\gamma}) /(\boldsymbol{1}+\boldsymbol{\gamma})] \boldsymbol{F X}
$$

L'équation (9) décrit le déficit du compte courant qui peut être soutenu sur le long terme si le ratio de la dette reste constant qu'on note $\mathrm{f}^{*}$ et le niveau des réserves désirée qu'on note $\mathrm{FX}^{*}$ augmentent en proportion de la croissance des importations :

$$
C A D=(\boldsymbol{\gamma}+\boldsymbol{\varepsilon}) \boldsymbol{f} *-[(\boldsymbol{\eta}+\boldsymbol{\varepsilon}-\boldsymbol{\gamma}) /(\mathbf{1}+\boldsymbol{\gamma})] \boldsymbol{F} \boldsymbol{X} * \quad(\mathbf{1 0})
$$

L'équation (10) suppose que l'économie pourrait désirer garder le ratio de réserves de changes aux importations constant. Par conséquent, les déficits courants durables varient selon les pays et dépendent des variables qui influent sur les décisions de portefeuille, ainsi que la croissance économique.

Une question non résolue est de savoir si l'investissement étranger direct net (IDE) devrait être inclus dans le calcul du niveau soutenable de la balance courante. Les IDE peuvent avoir un impact positif considérable sur les positions financières extérieures et, par conséquent, sur leurs perspectives de développement et ce en important de nouvelles technologies.

Les IDE peuvent améliorer la perception de la solvabilité de l'économie hôte, et ainsi réduire les couts d'emprunt et accéder à une gamme plus large de flux financiers. De plus il est maintenu que les IDE contribuent à l'augmentation des exportations et améliorent la balance courante sur le long terme. En intégrant les IDE dans le calcul du seuil de soutenabilité du compte courant au moyen terme on obtient d'après l'équation (10) :

$$
C A D=(\gamma+\varepsilon) f *-[(\boldsymbol{\eta}+\varepsilon-\gamma) /(1+\gamma)] F X *-i d e
$$


Il est à rappeler en dernier lieu que le calcul du déficit du compte courant par la méthode MFR est sujet à de sérieuses limitations. L'hypothèse de l'état stable de l'économie, constitue une hypothèse forte dans la mesure où les économies en transition comme le Maroc, des changements dans l'économie peuvent être prévisibles ou dans le cas où l'économie s'ajuste à un système d'ouverture sur le marché.

\section{Application pour le cas du Maroc}

Le calcul s'est basé sur la période 2007-2014. Dans nos calculs on a fixé le niveau désiré du ratio de la dette extérieure à $45 \%$ et le niveau des réserves de changes à la moitié du ratio d'importations. Quant au ratio de la dette extérieure, le taux de croissance réelle, le taux de change, les IDE et le taux de croissance des importations, sont pris en moyenne sur la période d'étude.

\section{Calcul du seuil de soutenabilité au moyen terme avec la méthode MFR}

Les hypothèses émanent de la littérature théorique et empirique de base du modèle de la méthode MFR, et sont calculés pour la période soit en moyenne arithmétique, soit en évolution géométrique pour la période 20072014. Les résultats sont présentées dans le tableau ci-dessous :

Tableau 1 Hypothèses du calcul du seuil de soutenabilité du compte courant sur le moyen terme

\begin{tabular}{|c|c|c|c|c|}
\hline $\begin{array}{c}\text { La dette } \\
\text { extérieure } \\
\text { en \% du } \\
\text { PIB }\end{array}$ & $\begin{array}{c}\text { Le taux d'intérêt } \\
\text { sur la dette } \\
\text { extérieure en } \% \\
(2013)\end{array}$ & $\begin{array}{c}\text { Le taux de } \\
\text { croissance réelle } \\
\text { en \% (2007-2013) }\end{array}$ & $\begin{array}{c}\text { La variation du } \\
\text { taux de change } \\
\text { en \% }(2007- \\
2013)\end{array}$ & $\begin{array}{c}\text { Les IDE en } \\
\% \text { du PIB } \\
(2007- \\
2013)\end{array}$ \\
\hline 21,9 & 3,2 & 3,9 & $-0,9$ & 4,1 \\
\hline
\end{tabular}

Pour évaluer la soutenabilité du compte courant sur le moyen terme on a utilisé la méthode MFR présenté ci-dessus en appliquant l'équation (4) dans les calculs. D'après la définition de la soutenabilité, la position du déficit du compte courant est toujours soutenable si les investisseurs sont toujours en mesure de le financer. Et puisque les IDE sont généralement perçus comme les flux financiers les plus stables, et la source de financement la plus importante après la dette au Maroc, on a appliqué l'équation (5) pour voir combien le déficit est financé par les IDE. Les résultats du calcul sont présentés ci-dessous :

Tableau 2 Seuils de soutenabilité du déficit du compte courant par la méthode MFR

Seuil de soutenabilité du compte courant par la méthode MFR en \% du PIB
Seuil de soutenabilité du compte courant par la méthode MFR en comptant les IDE en \% du PIB 
Pour évaluer la soutenabilité du compte courant marocain durant la dernière décennie on compare les seuils calculés au niveFau du compte courant actuel, comme présenté dans le graphe ci-dessous

Figure 1 le seuil de soutenabilité du compte courant calculé par la méthode MFR

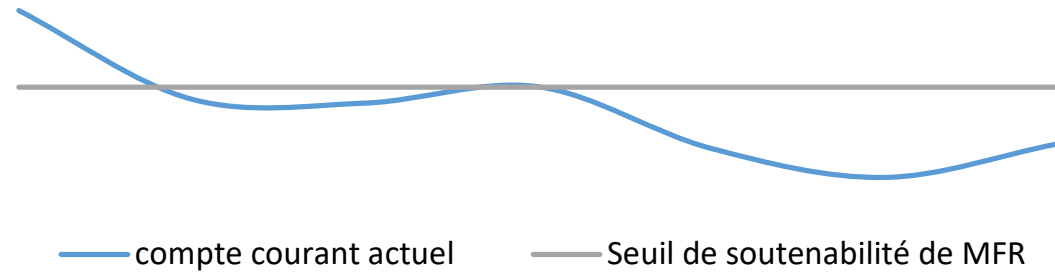

Le seuil calculé par la méthode MFR incluant les IDE est $-4,4 \%$ du PIB. L'évaluation de la soutenabilité du compte courant en se référant à ce seuil montre que la problématique de la viabilité extérieure s'impose avec la crise économique, la situation du compte courant marocain est insoutenable à partir de 2011.

Cependant sa position est moins inquiétante sur la période 2008-2010 où le compte courant effectif s'éloigne légèrement du seuil optimal affichant un écart maximal de 1,1 point en 2009 et revient à sa position optimale en 2010. C'est à partir de l'année 2011 que l'écart se creuse et atteint un niveau maximum de 5,3 points en 2012. Alors que le taux d'intérêt de la dette extérieure est resté relativement stable et bas durant toute la décennie (en moyenne $3 \%$ ), le taux de croissance relativement faible à partir de 2011 a causé la détérioration du compte courant.

Le seuil calculé sous l'hypothèse de l'arrêt brusque des entrées des IDE est très faible comparé au seuil calculé en intégrant les IDE. Dès lors on remarque que la situation du compte courant devient plus alarmante suite à un arrêt de revenus de capitaux étrangers sur le moyen terme. On constate encore une fois le rôle que jouent les IDE dans l'allègement du compte courant et dans son soutien pour le cas du Maroc.

\section{Calcul du seuil de soutenabilité avec la méthode Resein}

Les hypothèses du calcul du seuil de soutenabilité du compte courant sur le long terme sont présentées ci-dessous : 
Tableau 3 Hypothèses du calcul du seuil de soutenabilité du compte courant

\begin{tabular}{|c|c|c|c|c|c|c|}
\hline $\begin{array}{c}\text { La } \\
\text { moyenne } \\
\text { de dette } \\
\text { extérieure } \\
\text { en \% du } \\
\text { PIB (2007- } \\
2013)\end{array}$ & $\begin{array}{c}\text { La } \\
\text { variation } \\
\text { du taux de } \\
\text { change } \\
\text { réel } \\
\text { effectif sur } \\
\text { la période } \\
\text { (2007- } \\
2013)\end{array}$ & $\begin{array}{c}\text { La moyenne } \\
\text { du taux de } \\
\text { croissance } \\
(2007- \\
2013)\end{array}$ & $\begin{array}{l}\text { La moyenne } \\
\text { du taux de } \\
\text { croissance des } \\
\text { importations } \\
\text { réelles (2007- } \\
2013)\end{array}$ & $\begin{array}{c}\text { La } \\
\text { moyenne } \\
\text { des IDE } \\
\text { en \% du } \\
\text { PIB } \\
(2007- \\
2013)\end{array}$ & $\begin{array}{c}\text { La } \\
\text { moyenne } \\
\text { des } \\
\text { réserves } \\
\text { de } \\
\text { changes } \\
\text { en \%du } \\
\text { PIB } \\
(2007- \\
2013)\end{array}$ & $\begin{array}{c}\text { Le } \\
\text { niveau } \\
\text { désiré } \\
\text { des } \\
\text { réserves } \\
\text { de } \\
\text { change } \\
\text { en } \% \text { du } \\
\text { PIB } \\
(2007- \\
2013) \\
\end{array}$ \\
\hline 21,9 & $-0,9$ & 3,9 & 4,6 & 4,1 & 0,1 & 0,005 \\
\hline
\end{tabular}

Pour inclure le rôle des réserves de change désiré on applique la méthode de Reisen illustrée dans l'équation (11). Le calcul du seuil est fait en incluant les IDE, en supposant que le ratio de la dette toléré par les investisseurs est $45 \%$ et le niveau des réserves de change désiré est égal à la moitié du ratio des importations.

Le calcul dégage un seuil de déficit de 4,3\% du PIB. Le nouveau seuil calculé par la méthode de Reisen est plus serré que celui calculé par la méthode MFR. La soutenabilité du compte courant apparait dans le graphique cidessous :

Figure 2 le seuil de soutenabilité du compte courant calculés par la méthode de Reisen

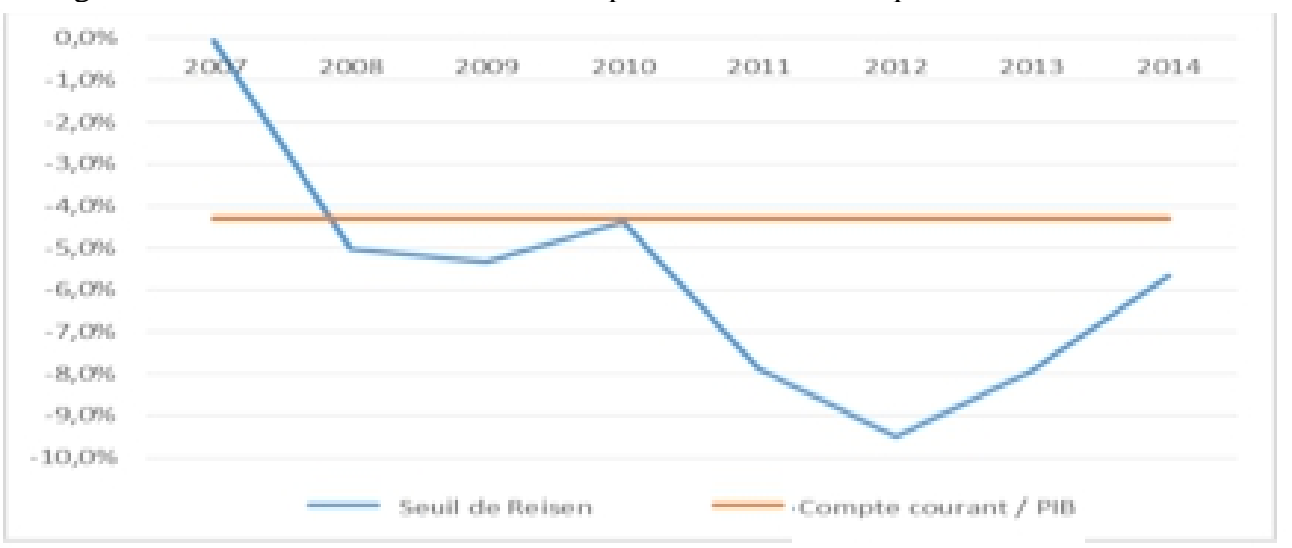

Le seuil optimal de soutenabilité sous l'hypothèse de la stabilité de la dette extérieure et des réserves de changes est de $-4,3 \%$ du PIB. En effet avec un ratio de la dette de $45 \%$, un ratio dont les investisseurs sont confiants que le pays en question réglera ses obligations et avec un niveau de réserves qui couvre la moitié des importations, la situation de la soutenabilité parait légèrement plus restrictive. 
A partir de 2011 même avec un flux d'investissement direct étranger moyen de $3,9 \%$ la position du compte courant parait insoutenable, conformément aux résultats affichés par la méthode de MFR où le déficit du compte courant était insoutenable tout au long de la période d'étude. Le déficit continue à se creuser affichant un écart de 5,4 points en 2012. Toutefois, cette tendance se renverse en 2013 lorsque le compte courant revient à un déficit de $-7,8 \%$.

Le Tableau 4 considère un ajustement hypothétique du ratio de la dette par rapport au PIB à $45 \%$ et un niveau des réserves souhaité qui couvre la moitié de nos importations. Le calcul s'est basé sur les projections annuelles des variables utilisées, en l'occurrence les projections du taux de change réel effectif, le taux de croissance et la dette extérieure de 2014 à 2018.

Le compte courant prévisionnel qui en résulte est de $-7,4 \%$ du PIB. C'est-à-dire que pour atteindre les niveaux de la dette au PIB et des réserves ciblées dans les cinq ans, le Maroc peut dégager un déficit du compte courant à une valeur de moins de 7,4\% sur le long terme.

Tableau 4 le seuil de soutenabilité du compte courant prévisionnel

\begin{tabular}{|c|c|}
\hline Equation utilisée & Résultat empirique \\
\hline \hline $\mathrm{CAD}=-1 / 5[\mathrm{f} * \mathrm{f}(1-\gamma-\varepsilon)-(\mathrm{FX}-(1-\eta+\varepsilon) /(1+\gamma) \mathrm{FX}]-\mathrm{IDE}$ & $-7,5 \%$ \\
\hline
\end{tabular}

L'étude de la soutenabilité du compte courant marocain met en évidence le rôle crucial des IDE dans le soutien du déficit courant. Le calcul du seuil de soutenabilité par les deux méthodes, donne des résultats identiques quant à l'évaluation de la situation de soutenabilité. Les hypothèses proposées par la méthode Reisen resserrent le seuil de soutenabilité, dans la mesure où les réserves de changes souhaitées ne couvrent que la moitié des importations et le seuil critique de la dette ne dépasse pas $45 \%$ du PIB.

Le compte courant marocain est insoutenable sur le moyen terme d'après les résultats des calculs effectués et ce à partir de 2008. Toutefois, la situation du compte courant ne devient inquiétante qu'à partir de 2011. Les perspectives de l'économie dans les 5 ans à venir, permettent un compte courant qui ne dépasse pas $7,5 \%$ du PIB en supposant une légère dépréciation du taux de change, et du taux de croissance du PIB prévues dans cette période.

Il est à rappeler que la position actuelle du compte courant renvoie à la persistance de son déficit, principalement la persistance du déficit commercial qui reflète une perte de compétitivité et une détérioration des équilibres extérieurs. Dans ce sens les autorités sont amenées à intervenir en matière de politique économique pour redresser la situation du compte courant. Cela ne peut se faire sans analyser les déterminants du compte courant pour dégager la source du déficit et ensuite analyser l'impact des variables explicatives sur le comportement futur du compte courant, ce qui fera l'objet de la partie qui suit. 


\section{Analyse empirique des déterminants du compte courant}

La plupart des travaux théoriques et empiriques sur les déterminants du solde courant sont basés sur l'approche inter-temporelle du compte courant, proposée par Sachs (1981) et Buiter (1981) et révisée par Obstfeld et Rogoff (1995). Selon cette approche, le solde courant est le reflet des décisions d'épargne et d'investissement, guidées par les orientations attendues de l'activité économique, les dépenses publiques, et divers autres facteurs économiques. La démarche consiste ainsi à identifier les variables qui affectent directement ou indirectement l'épargne et l'investissement.

\section{Spécification des variables retenues}

La sélection des variables susceptibles d'expliquer l'évolution du compte courant est inspirée de l'approche inter-temporelle. Dans notre modèle on s'est référé aussi à des variables liées aux facteurs externes susceptibles d'expliquer le comportement du compte courant Marocain. On classe alors les variables en deux catégories : Les variables liées au reste du monde et celles liées aux facteurs internes.

\begin{tabular}{|c|c|}
\hline Variables liées aux facteurs externes & Variables liées aux facteurs internes \\
\hline $\begin{array}{c}\text { Le taux d'ouverture : l'ouverture commerciale } \\
\text { est mesurée par la somme des exportations et } \\
\text { des importations par rapport au PIB. Elle } \\
\text { mesure le degré de l'ouverture économique au } \\
\text { commerce. La littérature empirique commune } \\
\text { prévoit une relation négative ou positive entre } \\
\text { l'ouverture commerciale et la balance du } \\
\text { compte courant selon que le pays a une base } \\
\text { d'exportations diversifiée ou non. } \\
\text { Le taux de change effectif réel : Le taux de } \\
\text { change effectif réel est un déterminant } \\
\text { fondamental du déficit du compte courant du } \\
\text { fait que son appréciation a tendance à stimuler } \\
\text { la demande d'importation et réduire la demande } \\
\text { extérieure pour les produits d'exportation. Le } \\
\text { signe attendu peut être positif ou négatif selon si } \\
\text { le compte courant dépend de l'élasticité à } \\
\text { l'import ou à l'export. }\end{array}$ & $\begin{array}{l}\text { Le taux d'épargne intérieure : Est une variable } \\
\text { clé selon l'approche inter-temporelle qui } \\
\text { considère que la balance du compte courant est } \\
\text { la différence entre l'investissement et } \\
\text { l'épargne. Selon Calderon (1999), une } \\
\text { augmentation du taux d'épargne publique ou } \\
\text { privé contribue à une baisse du déficit courant. } \\
\text { En effet, la hausse de l'épargne traduit en } \\
\text { général une baisse de la consommation qui } \\
\text { améliore le solde courant, à travers une } \\
\text { diminution des importations. Le signe attendu } \\
\text { est positif. } \\
\text { Le taux d'investissement : Comme l'épargne le } \\
\text { taux d'investissement est une variable décisive } \\
\text { dans le comportement du compte courant } \\
\text { d'après l'approche inter-temporelle. Un } \\
\text { accroissement des investissements détériore le } \\
\text { solde courant, un signe négatif est prévu. }\end{array}$ \\
\hline $\begin{array}{l}\text { Les flux d'investissements directs à l'étranger: } \\
\text { Certains auteurs se sont également intéressés } \\
\text { aux variables qui décrivent comment le déficit } \\
\text { courant est financé ou la façon dont l'excédent } \\
\text { du compte courant est utilisé sous forme } \\
\text { d'investissement certains à l'étranger. } \\
\text { L'accroissement des flux d'IDE contribue au } \\
\text { financement du déficit et permet un endettement } \\
\text { à moindres coûts grâce aux transferts de la }\end{array}$ & $\begin{array}{c}\text { Le taux de croissance : Comme déjà expliqué } \\
\text { dans la deuxième partie le signe attendu peut } \\
\text { être négatif ou positif selon les facteurs qui } \\
\text { induisent la croissance domestique. La relation } \\
\text { est négative lorsqu'une augmentation de la } \\
\text { croissance induit une augmentation de la } \\
\text { demande des produits étrangers entrainant une } \\
\text { hausse des importations et donc un creusement } \\
\text { du déficit courant. }\end{array}$ \\
\hline
\end{tabular}


technologie et l'amélioration de l'emploi. Le signe attendu est positif.

Les réserves de change: L'accumulation des réserves de change est une forme de financement du déficit courant. Plus un pays dispose d'un grand stock de réserves plus sa solvabilité augmente et peut s'endetter facilement et par conséquent financer son déficit courant. Le signe attendu est positif.

Le prix du pétrole: Pour les pays importateurs du pétrole (produit de première nécessité) comme le Maroc, une augmentation du prix du pétrole affecte directement la facture énergétique des importations, influant ainsi négativement le compte courant.

Tableau 5 liste des variables du modèle du compte courant

\begin{tabular}{|c|c|}
\hline Code & Description \\
\hline BCCR & Compte courant en pourcentage du PIIB \\
\hline TINV & Taux d'investissement \\
\hline TEP & Taux d'épargne \\
\hline TCROIS & Taux de croissance \\
\hline IDER & Investissements directs à l'étranger en pourcentage du PIB \\
\hline TOUV & Taux d'ouverture \\
\hline RESR & Ratio des Réserves de Change \\
\hline PET & Cours du pétrole \\
\hline
\end{tabular}

Estimation du modèle

\section{Méthodologie}

Dans cette étude on essaye d'expliquer la dynamique du compte courant par les variables choisies ci-haut. On utilise le modèle VECM pour capturer l'effet des déterminants du compte courant au LT. L'examen des coefficients de chaque variable ainsi que leur significativité permet de dégager les variables les plus pesantes dans le déficit de la balance courante.

Les données utilisées sont tirées de l'office des changes et de Bank Al Maghreb sauf le taux de change effectif réel qui est fourni par l'IFS. L'échantillon se base sur des données trimestrielles commençant du premier trimestre de 2004 au dernier trimestre de 2013, soit un total de 40 observations. La modélisation par VECM passe par plusieurs étapes, lesquelles on explicitera dans les paragraphes qui suivent.

\section{Test de stationnarité}

Pour mener un test de cointégration les séries testées doivent être intégrées du même ordre. On effectue le test de stationnarité des séries de variables choisies pour définir leur ordre d'intégration et ce grâce au test de 
racine unitaire AugmentedDickey Fuller. Mais avant tout il a fallu traiter la saisonnalité des séries puisque les données sont trimestrielles.

Mais avant tout il a fallu traiter la saisonnalité des séries puisque les données sont trimestrielles. Pour corriger les effets de saisonnalité on a utilisé la technique census X12 sous Eviews et on a procédé au test ADF dont les résultats figurent ci-dessous :

Tableau 6 Ordre d'intégration des variables du modèle du compte courant

\begin{tabular}{|c|c|c|c|c|}
\hline variable & Ordre d'intégration & Niveau & $\begin{array}{c}\text { Première } \\
\text { différence }\end{array}$ & $\begin{array}{c}\text { Seuil critique à } \\
5 \%\end{array}$ \\
\hline BCCR & $\mathrm{I}(1)$ & 0,045 & $-9,146$ & $-1,950$ \\
\hline TINV & $\mathrm{I}(1)$ & $-0,037$ & $-9,610$ & $-1,949$ \\
\hline TEP & $\mathrm{I}(1)$ & 0,715 & $-9,680$ & $-1,949$ \\
\hline TCER & $\mathrm{I}(1)$ & $-1,127$ & $-3,549$ & $-1,950$ \\
\hline TOUV & $\mathrm{I}(1)$ & 0,383 & $-4,733$ & $-1,949$ \\
\hline TCROIS & $\mathrm{I}(1)$ & $-0,769$ & $-6,182$ & $-1,951$ \\
\hline IDER & $\mathrm{I}(1)$ & $-0,270$ & $-7,839$ & $-1,950$ \\
\hline RESR & $\mathrm{I}(1)$ & $-1,298$ & $-5,866$ & $-1,949$ \\
\hline LPET & $\mathrm{I}(1)$ & $-0,918$ & $-4,870$ & $-1,950$ \\
\hline
\end{tabular}

Les résultats du test $\mathrm{ADF}$ en niveau, montrent que les statistiques de Studentcalculées sont toutes inférieures aux statistiques tabulées de DickeyFuller au seuil de 5\% ce qui montre que l'hypothèse nulle de l'existence d'une racine unité ne peut être rejetée. Il en ressort que les séries étudiées sont non stationnaires en niveau. Par contre les résultats obtenus en première différence montrent que les statistiques calculées sont inférieures aux valeurs critiques de Dickey-Fuller au seuil de 5\%. L'hypothèse nulle de la racine unité est donc rejetée au profit de l'hypothèse alternative du processus stationnaire. Les détails du test sont donnés en annexe 2 .

\section{Test de cointégration}

Après avoir mis en évidence la présence possible d'une racine unitaire dans les différentes séries considérées dans ce travail, on va tester maintenant, si ces variables sont cointégrées. Il s'agit d'étudier les interdépendances entre ces variables, sans faire d'hypothèse a priori sur la valeur des coefficients qui les relient et de tester l'existence des relations de long terme. On utilise la méthode de Johansen qui consiste en 3 étapes :

$>\quad$ La détermination du nombre de retards de la représentation VAR

$>\quad$ Le test de la trace et la détermination de la relation de long terme

$>\quad$ L'estimation du modèle à correction d'erreur 


\section{Détermination du nombre de retards}

La première étape de la démarche consiste à déterminer le nombre de retards de la représentation VAR. Le choix du nombre de retard à retenir dans le modèle a une importance particulière puisque, d'une part, ce dernier influence les estimations et, d'autre part, il est préférable d'avoir un petit nombre de retards puisque la taille de l'échantillon est réduite.

Tableau 7 Nombre de retards du modèle VAR

\begin{tabular}{|c|c|c|c|c|c|c|}
\hline \hline Lag & LogL & LR & FPE & AIC & SC & HQ \\
\hline & & & & & & \\
\hline 0 & -572.1475 & NA & 849.9739 & 32.28597 & 32.68185 & 32.42414 \\
\hline 1 & -363.1446 & 301.8930 & 0.793511 & 25.17470 & $29.13350^{*}$ & 26.55643 \\
\hline 2 & -249.2713 & $107.5471^{*}$ & $0.324519^{*}$ & $23.34840^{*}$ & 30.87012 & $25.97368^{*}$ \\
\hline \hline & & & & & \\
\hline
\end{tabular}

Le calcul des critères d'information LR, FPE, AIC, SC et HQ pour un maximum de retards égale à 2 montre que le retard optimal à utiliser dans l'estimation du modèle à correction d'erreur est 1 .

\section{Test de cointégration par la méthode de Johansen}

Une fois le nombre de retards à introduire dans le modèle VECM est fixé, l'étape suivante consiste à tester le nombre de relations de cointégration qui existent entre ces différentes variables.

Tableau 8 Résultats du Test de Johansen

\begin{tabular}{|c|c|c|}
\hline $\begin{array}{c}\text { Nombre de relations de } \\
\text { cointégrations }\end{array}$ & statistique de la trace & Valeur critique à 5\% \\
\hline Aucune & 325.5103 & 197.3709 \\
\hline Au plus 1 & 226.2808 & 159.5297 \\
\hline Au plus 2 & 160.3963 & 125.6154 \\
\hline Au plus 3 & 110.4444 & 95.75366 \\
\hline Au plus 4 & 72.45101 & 69.81889 \\
\hline Au plus 5 & 40.49756 & 47.85613 \\
\hline
\end{tabular}

Le test de la trace reporté dans le tableau indique l'existence de 4 relations de cointégration (hypothèse au seuil de $5 \%$ ) du fait que les statistiques de la trace sont supérieures à la valeur critique à 5\%. L'hypothèse nulle de l'absence de cointégration est rejetée. Ce résultat suggère que pendant la période (2004Q1-2013Q4) les séries sont cointégrées et convergent vers un équilibre de long terme. Dans notre étude on ne s'intéresse qu'à la relation de cointégration qui explique le ratio du solde compte courant à travers les autres variables explicatives. 


\section{Estimation du modèle vectoriel à correction d'erreur (VECM)}

Le choix de l'équation optimale a été fait en tenant compte des résultats des tests de significativité globale, du critère de R-squared, ainsi que de l'analyse des résidus de régression. Après plusieurs tentatives d'estimation de différents modèles on a opté pour le choix des variables présentées dans le modèle suivant dont la relation du long terme s'écrit :

$B C C R=0,135285 *$ TCROIS $-0,579638 *$ TINV $+0,789419 *$

TEP $-0,594706 *$ TCER $-5,811562 *$ LPET + 0,062247*IDER +

$0,071323 *$ RESR $-0,024538 *$ TOUV + 69,22790L'examen

$\mathrm{du}$

coefficient de correction d'erreur montre qu'il est significativement négatif, ce qui suggère l'existence d'une relation entre les variables au long terme. Les variables choisies expliquent bien le compte courant puisqu'elle sont significatives au long terme au seuil de $5 \%$ du fait que leurs T-statistiques sont supérieures à 1,96 sauf le taux d'ouverture.

Tableau 9 significativité des coefficients de la relation du long terme

\begin{tabular}{|c|c|c|}
\hline Variables & Coefficient & Significativité \\
\hline $\begin{array}{c}\text { Terme de correction } \\
\text { d'erreur }\end{array}$ & -1.090785 & $-2,26$ \\
\hline TCROIS & 0,135285 & $-2,25$ \\
\hline TINV & $-0,579638$ & 23,87 \\
\hline TEP & 0,789419 & $-17,66$ \\
\hline TCER & $-0,594706$ & 12,10 \\
\hline LPET & $-5,811562$ & 13,55 \\
\hline IDER & 0,062247 & $-1,71$ \\
\hline RESR & 0,071323 & $-9,57$ \\
\hline TOUV & $-0,024538$ & 0,86 \\
\hline
\end{tabular}

Les signes retrouvés dans le modèle sont en concordance avec les signes attendus dans la littérature économique discutée dans les paragraphes qui précèdent. Le taux de croissance a un effet positif sur le compte courant, les résultats montrent qu'un accroissement de la croissance du PIB de 1\% génère un surplus de $0,13 \%$ du ratio du compte courant.

Le taux d'investissement a un effet plus considérable sur la balance du compte courant dans la mesure où un accroissement de $1 \%$ du taux d'investissement réduit le compte courant de $0,57 \%$ en pourcentage du PIB. En effet le taux d'investissement s'est inscrit en hausse lors de la dernière décennie où il est passé de $27 \%$ en premier trimestre de 2004 à $33 \%$ en fin d'année 2013. Cette hausse s'est accompagnée d'une détérioration en ligne du compte courant.

Le taux d'épargne montre une relation positive avec le compte courant, une liaison logique du fait que l'épargne s'est détériorée durant la dernière décennie entrainant avec elle une détérioration du compte courant, le graphe ci-dessous montre que l'évolution des deux variables est identique dans le 
temps. D'autre part on remarque que le coefficient explicatif de la variable TINV est relativement haut. On constate que l'épargne est une variable déterminante dans l'explication du compte courant, son coefficient de corrélation aussi suggère le même résultat. Le calcul fait ressortir un coefficient de corrélation de 0,72 .

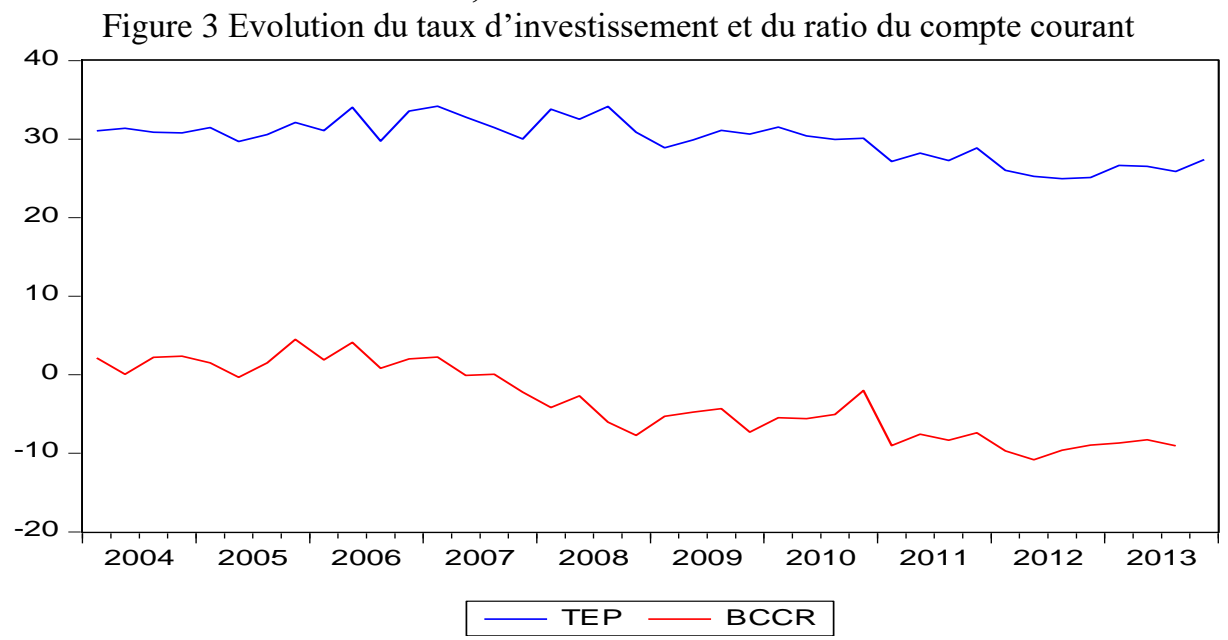

Comme prévu le TCRE a un impact négatif sur le compte courant. Une appréciation de ce dernier de $1 \%$ détériore la balance courante de $0,59 \%$ et rend nos importations plus couteuses. En Outre la significativité du coefficient du TCER est forte, ce qui suppose le rôle d'une politique de taux de change qui peut alléger le déficit du compte courant.

Le prix du pétrole est la variable la plus pesante dans le déficit courant d'après le modèle suggéré. L'influence négative du prix du pétrole s'explique par le fait que le Maroc est un pays importateur du pétrole, en effet l'importation des produits pétroliers constitue 24\%de l'ensemble des importations en 2013, contribuant avec grande ampleur au déficit commercial. Le Maroc reste donc dépendant à la conjoncture extérieure puisque ses importations de produits énergétiques ne cessent de s'alourdir.

Les IDE et les réserves de changes impactent positivement et significativement le compte courant. Le Maroc a pu attirer plus d'investissement étrangers durant la dernière décennie, bénéficiant de la rentée de devises et de l'importation de la technologie qui joue en faveur de la croissance et donc de l'amélioration du compte courant. Les réserves de changes quant à eux permettent de financer le déficit du compte courant de $0,07 \%$ lorsque l'accumulation des réserves de change augmente de $1 \%$.

Le taux d'ouverture est lié négativement au compte courant. En effet malgré l'ouverture du Maroc sur de nouveaux marchés, il n'a pas bénéficié d'une prolifération de la balance commerciale, dans la mesure où le taux de couverture s'est dégradé. L'ouverture a donc pénalisée les exportations au 
profit des importations. Toutefois, l'impact de cette variable n'est pas significatif dans le long terme dans le modèle suggéré.

Au terme de cette étude on constate le rôle majeur des facteurs internes dans l'explication du déficit du compte courant et de la vulnérabilité du pays aux prix mondiaux du pétrole. Il en est ressortit de l'estimation des coefficients des variables explicatives, que la variable la plus pesante est le prix du pétrole du fait que les importations des produits énergétiques sont classées en premier rang.

Ensuite viennent le taux d'épargne suivi par le taux d'investissement, deux variables réelles qui agissent directement sur le compte courant à travers la demande intérieure. En outre l'impact négatif du taux de change réel est aussi considérable. Finalement on a remarqué que l'effet des IDE et les réserves de change n'est pas négligeable, une augmentation de ces deux moyens de financement améliore le compte courant.

\section{Tests de validité du modèle}

Plusieurs statistiques de tests ont été calculées afin d'apprécier la qualité de l'estimation (le test Box-Pierce d'autocorrélation des résidus, et le test de normalité des résidus de Jarque-Bera). Ces tests constituent un moyen de détecter la défaillance éventuelle de certaines hypothèses effectuées lors de l'estimation du modèle. Les résultats de ces tests révèlent que les principales hypothèses faites sur les résidus sont satisfaites par le modèle suggéré.

Figure 4 Test de Box-Pierce

\begin{tabular}{|c|c|c|c|c|c|c|c|c|}
\hline \multicolumn{2}{|c|}{ Autocorrelation } & \multicolumn{3}{|c|}{ Partial Correlation } & \multirow{2}{*}{$\frac{A C}{0.017}$} & \multirow{2}{*}{$\frac{\text { PAC }}{0.017}$} & \multirow{2}{*}{$\frac{\text { Q-Stat }}{0.0111}$} & \multirow{2}{*}{$\frac{\text { Prob }}{0.916}$} \\
\hline I & I & 1 & 1 & 1 & & & & \\
\hline 14 & 1 & 1 마 & 1 & 2 & -0.173 & -0.174 & 1.2180 & 0.544 \\
\hline & 1 & 1 & 1 & 3 & -0.086 & -0.082 & 1.5248 & 0.677 \\
\hline & 1 & & 1 & 4 & 0.088 & 0.062 & 1.8550 & 0.762 \\
\hline 15 & 1 & 19 & 1 & 5 & -0.065 & -0.099 & 2.0386 & 0.844 \\
\hline 1 무 & 1 & 1 다 & 1 & 6 & -0.153 & -0.140 & 3.1109 & 0.795 \\
\hline 14 & 1 & 1 . & 1 & 7 & -0.086 & -0.104 & 3.4605 & 0.839 \\
\hline 1 & 1 & 1 & 1 & 8 & 0.167 & 0.107 & 4.8279 & 0.776 \\
\hline 14 & 1 & 1 다 & 1 & 9 & -0.074 & -0.134 & 5.1084 & 0.825 \\
\hline 14 & 1 & 14 & 1 & 10 & -0.106 & -0.077 & 5.6978 & 0.840 \\
\hline 1 다 & 1 & 1 마 & 1 & 11 & -0.127 & -0.163 & 6.5769 & 0.832 \\
\hline 1 & 1 & 14 & 1 & 12 & 0.005 & -0.108 & 6.5784 & 0.884 \\
\hline 1 & 1 & 1 & 1 & 13 & 0.209 & 0.167 & 9.1832 & 0.759 \\
\hline 1 & 1 & 1 & 1 & 14 & -0.014 & -0.051 & 9.1955 & 0.818 \\
\hline 1 - & 1 & 15 & 1 & 15 & -0.105 & -0.080 & 9.9101 & 0.825 \\
\hline 1 & 1 & 1 다 & 1 & 16 & -0.034 & -0.112 & 9.9898 & 0.867 \\
\hline
\end{tabular}

Les p-value du test de Box-Pierce sont toutes supérieurs à 0,05 . On déduit alors que le résidu du modèle VECM choisi est un bruit blanc. Finalement le test de Jarque-Bera montre que les résidus sont distribués normalement car la probabilité affiche une valeur supérieure à 0,05 . 
Figure 5 Test de normalité des résidus

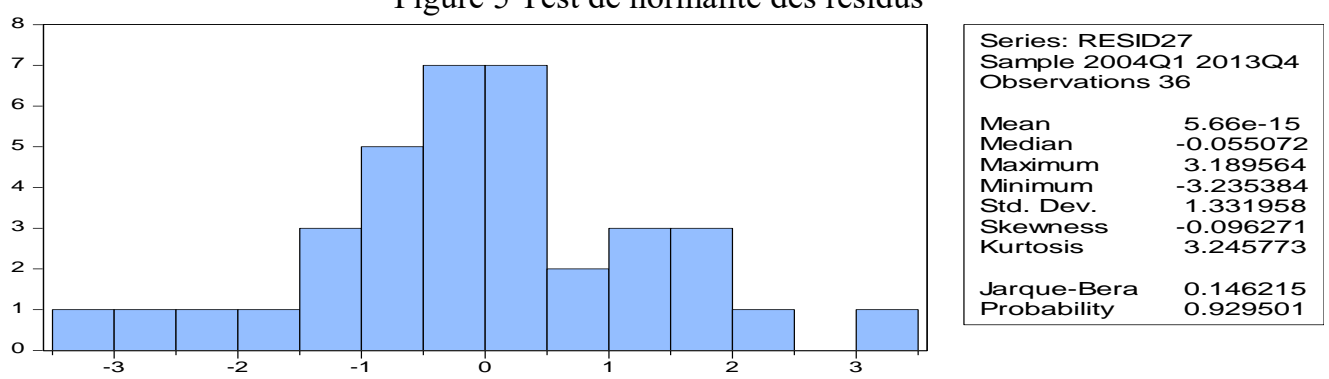

\section{Analyse des réponses Impulsionnelles}

Une fois le modèle VECM spécifié, il peut être utilisé pour appréhender les relations dynamiques existant entre les 9 variables du modèle. Pour cela, on aura recours à l'analyse des fonctions de réponses aux chocs exercés par les variables explicatives sur le compte courant. Le sens d'évolution des fonctions de réponses est en ligne avec le signe des variables déjà discuté. En outre la fonction de réponse du compte courant au choc des variables internes n'est pas stagnante, ce qui signifie que le comportement du compte courant est fortement affecté par ces variables, chacune selon son poids dans le modèle.

L'analyse des graphiques ci-dessous montre qu'en stimulant le taux d'épargne et le taux de croissance, le compte courant répond positivement à ce choc et affiche des déficits moins importants. En effet, si on augmente le taux d'épargne de $1 \%$, le compte courant augmente de $0,6 \%$ en moyenne sur le moyen-terme, plus précisément dans la période de six trimestres. La promotion de la croissance joue aussi en la faveur de l'allègement du déficit courant, en effet une augmentation de $1 \%$ du taux de croissance améliore le solde du compte courant sur une période de 6 trimestres allant de $0,5 \%$ à $0,2 \%$. 
Figure 6 Fonctions de réponses impulsionnelles des variables internes

Response to Generalized One S. D. Innovations Response of BCCR to TINV
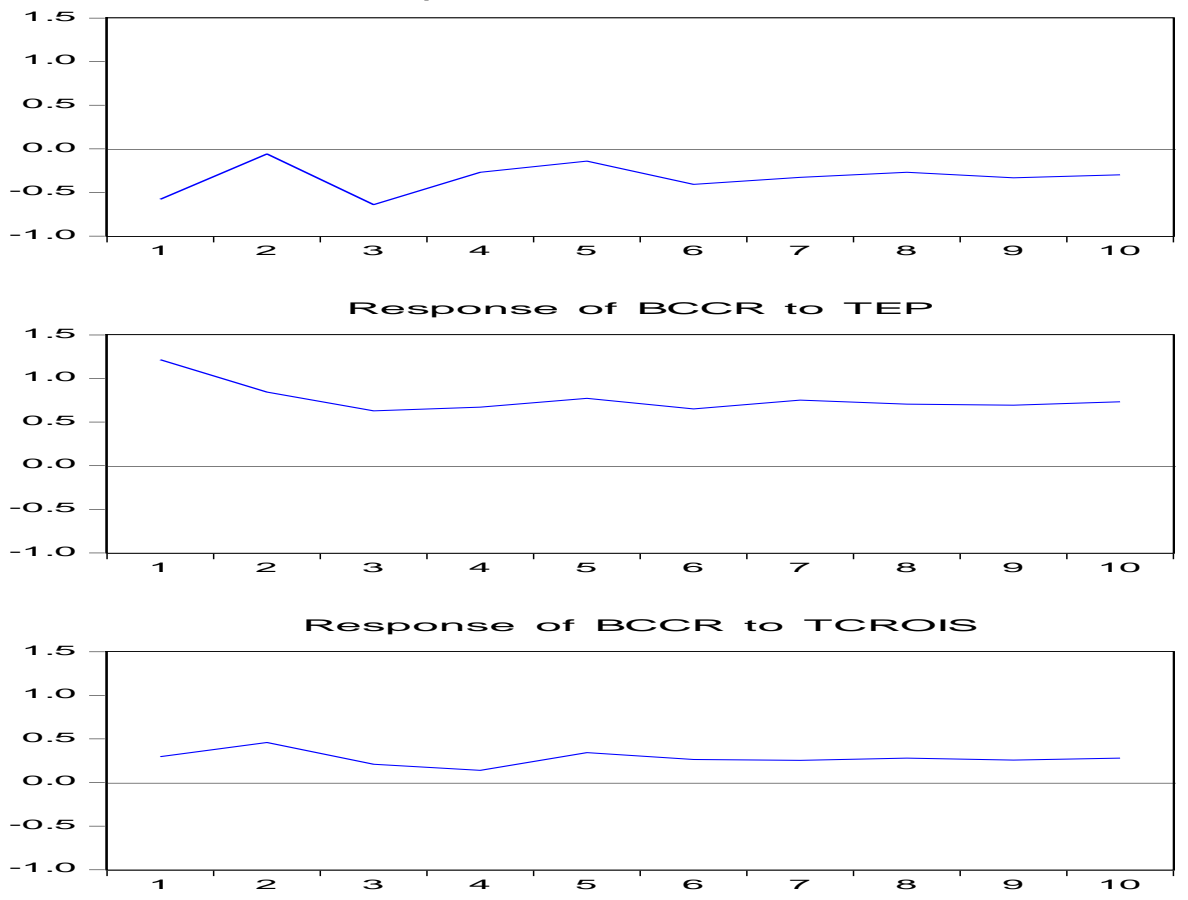

L'évolution du compte courant est sensible au choc du prix du pétrole, dans le sens où ce dernier détériore le solde courant de $6 \%$ suite à une augmentation de $1 \%$ et ce dans une période de 10 trimestres. On conclue que si le Maroc ne réduit pas sa consommation énergétique il sera toujours dépendant des cours pétroliers mondiaux qui ne cessent de flamber.

Les IDE améliorent le solde du compte courant dans le court et moyen terme, un choc positif augmente le solde courant de 1\%. Dans ce sens, la politique économique doit s'orienter vers l'attraction des IDE qui constituent un grand potentiel qui n'est pas exploité totalement par le Maroc. L'impact du taux de change sur le compte courant est pratiquement éphémère, du fait de sa rigidité. Une dépréciation du taux de change aurait pu améliorer le compte courant, cela dit, la politique de change fixe devrait être revisitée.

Les réserves de change impactent positivement le compte courant de l'ordre de 1\%. Dans les 10 trimestres futurs, si le Maroc parvient à augmenter ses réserves de change de $1 \%$ son compte courant s'améliorera de $1 \%$ aussi, un impact assez important puisque l'accumulation les réserves de changes permettra de garder la solvabilité du compte courant. On conclue donc que le Maroc doit s'investir plus dans la promotion des exportations, l'attraction des IDE et la promotion du tourisme en vue d'accumuler plus de réserves de change. 
Figure 7 Fonctions de réponses des variables externes Accumulated Response to Generalized One S.D. Innovations

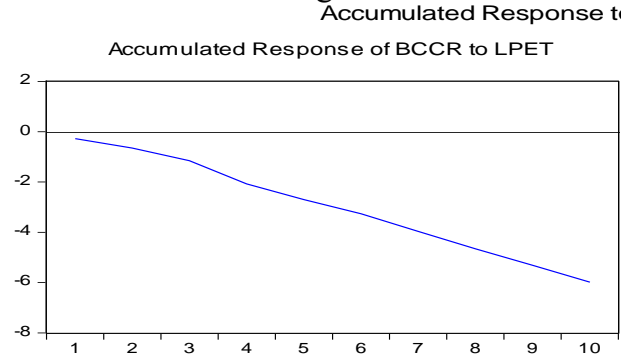

Accumulated Response of BCCR to RESR
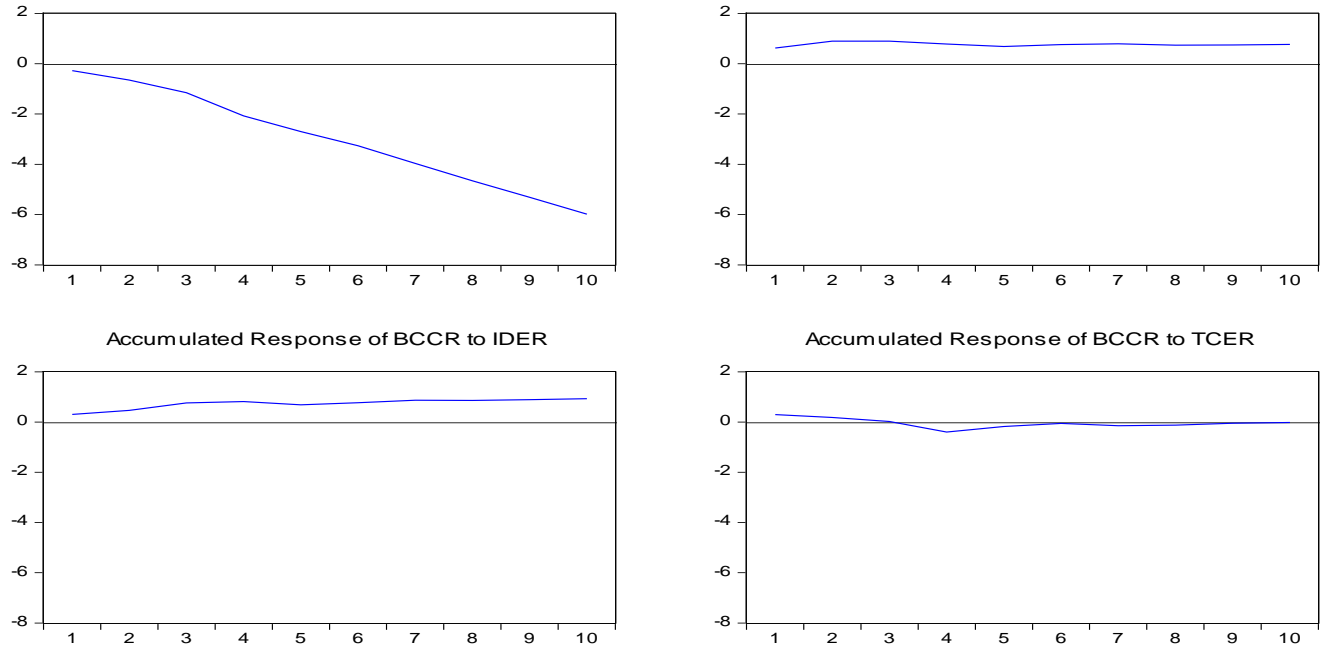

Accumulated Response of BCCR to TCER

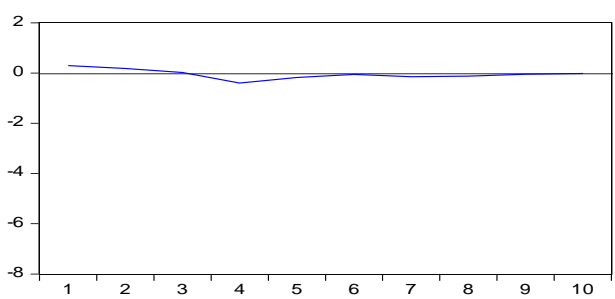

\section{Conclusion}

Le processus d'intégration du Maroc dans l'économie mondiale exige une grande disposition à l'adaptation aux normes standards régulant le marché international. Il est donc nécessaire pour le Maroc de préparer une plateforme adéquate afin d'assouplir la transition vers l'ouverture et limiter les effets néfastes qui pourraient en résulter. L'ouverture économique du Maroc a suscité la mise en place de plusieurs politiques notamment monétaires. En effet, pour accompagner cette réforme stratégique, les politiques publiques ont été appelées à répondre à une exigence précise qui est la mise en place des mesures pour pouvoir mettre à niveau les secteurs économiques.

Ainsi, les réformes économiques engagées dans les dernières années visent-elles principalement le renforcement de la diversification économique et de la compétitivité dans le but de stimuler la production et les exportations. En effet, les performances économiques du pays s'appuient sur les nouvelles politiques macroéconomiques adoptées, les efforts fournis pour la soutenabilité de la Balance des Paiements.

L'examen du seuil de soutenabilité calculé par la méthode de Reisena montré conformément aux résultats de MFR que les signes d'insoutenabilité apparaissent depuis 2008. Cependant, ce n'est qu'en 2011 que le compte courant affiche des déficits qui dépassent largement le seuil optimal exposant l'économie nationale à une plus grande vulnérabilité.

Ainsi, l'analyse économétrique effectuée, montre que le compte courant dépend de variables internes comme le taux de croissance, le taux d'investissement et le taux d'épargne aussi bien des variables liées aux facteurs 
externes comme le taux de change, le prix du pétrole, les IDE, les réserves de changes et le taux d'ouverture.

L'estimation du modèle du compte courant proposé, suggère qu'au long terme, le prix du pétrole est la variable la plus déterminante sur le compte courant, suivie du taux d'épargne, le taux de change et aussi le taux d'investissement. Enfin l'examen des réponses impulsionnelles, nous éclaire sur les opportunités dont dispose le Maroc pour alléger ces déficits, notamment le développement d'une infrastructure apte à attirer plus d'IDE qui jouent un double rôle, d'une part, le financement du déficit courant et d'autre part, l'importation des technologies qui jouent en faveur de l'amélioration de la croissance économique.

Par ailleurs, l'accumulation des réserves de change participe aussi avec une grande part dans l'allègement du déficit. Le Maroc est amené à diversifier sa base de produits à l'export qui sont à faible valeur ajoutée et à créer un climat propice d'investissement pour attirer plus d'IDE. D'autre part, le poids alarmant de la facture énergétique dans les dépenses de l'état, et la dépendance de nos importations aux cours mondiaux du pétrole, l'incite à s'orienter vers l'investissement dans les énergies renouvelables.

\section{References:}

1. Aghevli Bijan, J.M., Bouhton, P.J., Montiel, D., Villanueva, \& Woglom, G. (1990). "The role of the National Saving in the World Economy:" Recent Trends and Prospects", IMF Occasional Paper 67. IMF, Washington, D.C.

2. Bank Al-Maghrib. Rapports annuels, 1980-2012 Bank Al-Maghrib, Rabat, Maroc.

3. Banque mondiale (2010). "Maroc: Sources de croissance". Washington, D.C.

4. Barro, Rober, J. (1987). “La Macroéconomie’', Éditions Armand Colin, Paris

5. Benmansour, A. (1994). "Un exemple de réussite: le Maroc', Revue d'Economie Financière (hors série). Paris France.

6. Bouhga-Hagbe, J. (2004). "On the Long-Term Determinants of Workers Remittances in Morocco". Morocco: Selected Issues.Country Report No. 04/164. IMF, Washington, D.C.

7. Calderon, C. \& Loayza. (2009). "Determinants of Current Accounts in Developing Countries", Working Papers Central Bank of Chile 51, Central Bank of Chile.

8. Clark, P., Bartolini, L., Bayoumi, T., \& Symansky, S. (2004). "Exchange Rates and Economic Fundamentals: A Framework for Analysis." IMF Occasional IMF, Washington, D.C. 
9. Direction du Trésor et des Finances Extérieures, (1988). Compte-rendu de la réunion du 16-11-88 relative à la politique du rapatriement des TME. Ministère des Finances.

10. Fonds monétaire international, (2012). "Maroc: Evolution récente de l'économie, SM/82/205'. Washington, D.C.

11. Hinkle, L.E. \& Montiel, P. J. (eds.) (1999). "Exchange Rate Misalignment, Concepts and measurements for developing countries". New York: Oxford University Press.

12. Isard, P., Faruqee, H., Kincaid, G.R., \& Fetherston, M.J. (2001). "Methodology for Current Account and Exchange Rate Assessments". IMF Occasional Paper No. 209. IMF, Washington, D.C.

13. Takatoshi, I. (1998). "What Can Developing Countries Learn from East Asia's Economic Growth" Annual World Bank Conference on Development Economics, Washington.

14. Khan Mohsin, S. \& Senhadji Abdelhak, S. (2000). "Thresholds Effects in the Relationship between Inflation and Growth". IMF Working Paper WP/00/110. IMF, Washington, D.C.

15. Knight Malcolm \& Fabio Scacciavillani (1998). "Currents Accounts: What is their Relevance for Economic Policymaking?". IMF Working Paper WP/98/71. IMF, Washington, D.C.

16. Lane Philip \& Gian Maria Milesi-Ferretti (1999). "The External Wealth of Nations - Measures of Foreign Assets and Liabilities for Industrial and Developing Countries ". IMF Working Paper WP/99/158. IMF, Washington, D.C.

17. Lane Philip \& Gian Maria Milesi-Ferretti (2000). " The Transfer Problem Revisited: Net Foreign Assets and Real Exchange Rates". IMF Working Paper WP/00/123. IMF, Washington, D.C.

18. Little, I.M.D. \& Richard, NC. (1993). "Boom, Crisis, And Adjustment: The Macroeconomic Experience of Developing Countries'. Oxford University Press, the World Bank.

19. Maciejewski Edouard, B. (1983). "Real Effective Exchange Rate Indexes: A Re-examination of the Major Conceptual and Methodological Issues." IMF Staff Papers 30(3): 491-541. IMF, Washington, D.C

20. Milesi-Ferretti, G.M. \& Razin, A. (1996). "Current Account Sustainability". Princeton Studies in International Finance $n^{\circ} 81$. Princeton, New Jersey.

21. Polak, J. (2001). "The two monetary approaches to the balance of payments: Keynesian and Johnsonian." IMF Working Paper WP/01/100. IMF, Washington, D.C. 
22. Sarr, A. (2004). "Assessing the Sustainability of Public Debt in Morocco". Morocco: Selected Issues. Country Report No. 04/164. IMF, Washington, D.C. 